\title{
Thermal processing effects in proton-isolated n-type GaAs devices
}

\author{
$\underline{\text { S. Ahmed }{ }^{*^{1}}}$, K. Amirov ${ }^{2}$, U. Larsson ${ }^{2}$, P. Too ${ }^{1}$, B. J. Sealy ${ }^{1}$ and R. Gwilliam ${ }^{1}$ \\ ${ }^{1}$ Advanced Technology Institute, University of Surrey, Guildford, GU2 7XH, United Kingdom \\ ${ }^{2}$ Department of Physics, Uppsala University, Box 530 SE-751 21 Uppsala, Sweden \\ *Tel: 44 (0) $1483683373 \quad$ Fax: 44 (0) $1483689404 \quad$ E-mail: s.ahmed@eim.surrey.ac.uk
}

\begin{abstract}
In this paper we present the results of two new experiments (1) Infrared Reflectivity (IR) measurements of the thickness of the modified layers following the proton implantation in n-type GaAs layers at various implant temperatures and (2) a detailed study of the thermal stability of n-type GaAs layers isolated by proton implantation. It is found that the threshold dose (minimum implant dose required for a maximum sheet resistivity) for a device is a key parameter to obtain good thermal stability. The optical properties also depend on the implantation temperature and dose.
\end{abstract}

\section{Introduction:}

Compound semiconductors are currently employed in the fabrication of optoelectronic devices and integrated circuits (IC) for high frequency operations [1]. Ion bombardment with appropriate ion doses in these materials is used to convert a conductive layer to a highly resistive one or to improve the electrical isolation between neighbouring devices in ICs. Electrical isolation of III-V semiconductors formed by introduction of a controlled concentration of point defects from light ion irradiation is a well-established technique [2]. Besides the efficiency of this method to obtain highly isolated layers, it has the additional advantage of maintaining the planarity of the isolated structures [3]. Proton implantation of the n-type GaAs wafers produces a variety of lattice defects, such as vacancies and interstitials, which can trap the free electrons and reduce the carrier concentration in the implanted region. Thermal processing of the crystal tends to remove this damage and reduce the degree of compensation, while at the same time producing diffusion of the defect centres [4]. Proton implantation is also used to form infrared optical waveguides as well as other guided wave components [4]. For such devices, annealing of the implanted regions is required in order to reduce the propagation losses to acceptable levels [4-5]. Therefore thermal processing due to elevated implantation temperature and post-implant annealing cycles is crucial to enable electrical and optical characteristics. In the present article, a systematic investigation of the dependence of the threshold dose on the thermal process for isolation as a function of the original donor concentration in GaAs structures and their thermal stability is reported. Optical changes due to variation of the implantation temperature are also discussed.

\section{Experimental:}

The starting material was prepared using multi-energy silicon ion implantation into semi-insulating GaAs at various doses to form a micron thick n-type GaAs layer of two different initial sheet carrier concentrations $\left(n_{\mathrm{s}}=3.3 \times 10^{13} \mathrm{e}^{-/} / \mathrm{cm}^{2}\right.$ and $\left.n_{\mathrm{s}}=3.2 \times 10^{14} \mathrm{e}^{-/} / \mathrm{cm}^{2}\right)$. The isolation was obtained by proton irradiation at energy of $300 \mathrm{keV}$ to place the peak of the 
damage distribution ( 2.45 microns) well inside the semi-insulating GaAs substrate and to produce a constant level of damage in the near surface region. All doping implants were performed at room temperature using a Danfysik 1090 implanter or a 2MV High Voltage Engineering Europe implanter. The normal to the wafers was inclined at $7^{\circ}$ with respect to the beam to minimise ion channelling. The wafers were then encapsulated on both back and front sides with $500-800 \AA$ of $\mathrm{Si}_{3} \mathrm{~N}_{4}$ deposited by the plasma enhanced chemical vapour deposition (PECVD) technique at a temperature of $300^{\circ} \mathrm{C}$. Rapid thermal annealing (RTA) was carried out at $900^{\circ} \mathrm{C}$ for $30 \mathrm{~s}$ in order to electrically activate the implanted $\mathrm{Si}$. The encapsulant was removed in $10 \%$ buffered HF acid and cleaned with organic solvents after being cleaved to obtain several samples of approximately $1 \mathrm{~cm}^{2}$. Cloverleaf patterns were photo-lithographically defined on the surface of the samples using photoresist. Following this process, the exposed area of the samples was etched to a depth of $4 \mu \mathrm{m}$ in a solution of $\mathrm{H}_{2} \mathrm{O}_{2}$. $\mathrm{H}_{2} \mathrm{SO}_{4}: \mathrm{H}_{3} \mathrm{PO}_{4}$ (1: $2.5: 2.5 \mathrm{vol}$.) to form structures suitable for van der Pauw measurements. The total etch depth was measured using a Rank Taylor Hobson Talystep, the error typically being $5 \%$. Ohmic contacts were formed using tin $(\mathrm{Sn})$ dots applied to the GaAs surface and annealed for 1-2 minutes in a forming gas ambient. Isolation implants were performed at different doses $\left(5 \times 10^{13} \mathrm{~cm}^{-2}, 2 \times 10^{14} \mathrm{~cm}^{-2}, 5 \times 10^{14} \mathrm{~cm}^{-2}\right.$ and $\left.2 \times 10^{15} \mathrm{~cm}^{-2}\right)$ at room temperature (RT). Additionally, some samples were also implanted at $-196^{\circ} \mathrm{C}, 100^{\circ} \mathrm{C}$ and $200^{\circ} \mathrm{C}$ for optical characterisation using Infrared Reflectivity measurements. For liquid nitrogen temperature $\left(-196^{\circ} \mathrm{C}\right)$ and hot implantation, special arrangements were made in order to cool down or heat up the sample stage to the required temperatures. During implantation the beam current was maintained below $0.1 \mu \mathrm{Acm}^{-2}$. The doped regions under the contact areas were masked using the previously applied Sn contacts and a molybdenum foil on top of those contacts.

The Hall and resistivity measurements were carried out using a Bio-Rad HL5900 system. All measurements were done at RT. The reflectivity spectra of the samples were taken with a Shimadzu UV3010 spectrophotometer. The beam was incident at an angle of 30 degrees from normal with a spread of \pm 8 degrees. Measurements were made over a spectral range from 4000 to $180 \mathrm{~cm}^{-1}$ and a number of fringes were observed for each of the as-implanted samples. The fringe patterns in the range from 2000 to $600 \mathrm{~cm}^{-1}$ were very periodic and hence were used to determine the thickness of the layer.

\section{Results and Discussion:}

\section{Infrared Reflectivity (IR) Measurements}

Monochromatic light reflected from a transparent film on a substrate displays interference effects due to multiple reflections at both boundaries of the film [5]. Many properties of the film including its thickness and refractive index, can, in principle, be determined from this set of fringes. If the refractive index of the sample $\eta_{\mathrm{s}}$ is uniform and does not vary appreciably over the spectral range of measurement, the resulting fringe pattern will be simply periodic [6]. The periodicity of the fringes resulting from the Infrared Reflectivity measurements can be used to estimate the thickness of the compensated film, which is inversely proportional to the average fringe separation. To a good approximation thickness, $t_{1}$ is given by [7]:

$$
t=\frac{1}{2 S\left(\eta_{s}^{2}-\sin ^{2} \theta\right)^{1 / 2}}
$$


where $S$ denotes the average spectral separation (in wavenumbers) and $\theta$ is the angle of incidence of the incoming light. A value of 3.29 was used for $\eta_{\mathrm{s}}$ since this is the average refractive index for intrinsic GaAs in the specified spectral range [5]. Figure 1 shows the measured average fringe separation as a function of implantation temperature for protonisolated GaAs layers of initial sheet carrier concentration of $3.3 \times 10^{13} \mathrm{~cm}^{-2}$. The measurements were performed for a range of proton doses at different implantation temperatures.

Liquid Nitrogen implants are shown (Figure 1) to yield the lowest average fringe separation and hence the thickest optically modified layer at each of the fluence levels used in these experiments. For each substrate temperature during implantation, the thickness of the compensated layer increased with increasing ion dose. For example, for a higher dose of $2 \times 10^{15} \mathrm{~cm}^{-2}$ the cryogenic implants produce a compensated layer of about 4.1 microns thick whereas the thickness is 3.3 microns in the case of $200^{\circ} \mathrm{C}$ implants. For a relatively lower dose of $5 \times 10^{13} \mathrm{~cm}^{-2}$ the thickness of compensated layer is about 3.2 and 2.8 microns, respectively for liquid nitrogen and $200^{\circ} \mathrm{C}$ implants. It is interesting to note that the original $\mathrm{n}$ type layer is only about a micron thick for these experiments. The projected range, $R_{p}$, and straggle, $\delta \mathrm{R}_{\mathrm{p}}$, of $300 \mathrm{keV}$ protons in $\mathrm{GaAs}$ are 2.45 and 0.27 microns, respectively as determined by the simulation code Transport of Ions in Matter (TRIM) [8,11]. We observe that $200^{\circ} \mathrm{C}$ implants with lower proton dose produces a 2.8 microns thick compensated layer, which is in agreement with the thickness $\left(R_{p}+\delta R_{p}\right)$ calculated by TRIM. For further higher doses, the compensated layer produced at $200^{\circ} \mathrm{C}$ implantation is thicker than the calculated thickness. If the defect centres are primarily removed during the enhanced dynamic annealing due to hot implantation, the thickness of the compensated layer is reduced. The extent of this reduction may vary with the extent of damage produced by the proton implantation at various doses. Further analysis of the reflectivity data is expected to yield accurate values for the depths to which the optical effects extend.

\section{Electrical Measurements}

Samples implanted at RT were subsequently annealed in order to observe any changes in sheet resistivity $\left(R_{\mathrm{s}}\right)$ with the post-implanted annealing temperatures. The thermal stability of implant isolation is a critical issue to consider in device applications. In particular, maximising the thermal stability of the isolated regions is desirable. It is known [8-10] that the electrical isolation depends critically on ion dose and initial free carrier concentration. The dose dependence of sheet resistivity for our material [8] shows that at the low doses, $R_{s}$ remains relatively unaffected, while it increases abruptly due to both free carrier trapping at defect levels and to mobility degradation with increasing dose. The sheet resistance as a function of dose reaches a narrow plateau when the concentration of deep levels is of the same order of magnitude as the free carrier concentration [9]. Any further increase in dose beyond this plateau results in the monotonic decrease in $R_{s}$ because of hopping conduction of carriers between closely spaced defects [9]. Following Ref. [8], a threshold dose for isolation, $D_{\text {th }}$, can be defined as the minimum ion dose at which $R_{s}$ reaches its maximum value. These results [810] show that ion doses required for an effective isolation of GaAs have simple linear and reciprocal dependences on the values of initial free carrier concentration and ion-beamproduced atomic displacements, respectively. As a result, implant conditions necessary for an effective electrical isolation of a particular GaAs-based device can be readily estimated based on experimental data and the number of atomic displacements calculated using, for example, the TRIM code [11]. If $n_{s o}$ is the original sheet carrier concentration of the n-type GaAs layers, $D$ is the implanted dose to isolate the structure, $n_{S D}$ is the measured sheet carrier concentration after the isolation implantation to dose $D$ then $R$ will be the carrier removal rate which is a 
function of mass and energy of the ion. The equation relating these parameters is as follows [8]:

$$
n_{s o}=n_{s D}+R \times 1
$$

Based on the above model, it is estimated that an average number of seven protons with energy of $300 \mathrm{keV}$ are required to compensate one electron. The threshold doses in this experiment are (using the results mentioned above [8]) $D=2 \times 10^{14} \mathrm{~cm}^{-2}$ and $D=2 \times 10^{15} \mathrm{~cm}^{-2}$ for the n-type GaAs layers with initial sheet carrier concentrations of $3.3 \times 10^{13} \mathrm{e}^{-1} / \mathrm{cm}^{2}$ and $3.2 \times 10^{14} \mathrm{e}^{-/ \mathrm{cm}^{2}}$, respectively. Figures 2 and 3 illustrate the resistivity of the isolation for layers implanted with protons at RT for a range of doses including the threshold dose. Each data point is comprised of the cumulative isochronal annealing sequence for 60 s periods at the lower RTA temperatures. For the $D_{\text {th }}$ case in figure 2, the stability of the isolation is restricted to temperatures below $400{ }^{\circ} \mathrm{C}$. Above $400{ }^{\circ} \mathrm{C}$ a decrease of $R_{\mathrm{s}}$ by 4 orders of magnitude is observed. The decrease of the dose from $D_{\text {th }}$ to $0.25 D_{\text {th }}$ resulted in a remarkable decrease in the thermal stability above $100^{\circ} \mathrm{C}$, although the as implanted resistivity value is as high as $\sim 10^{7} \mathrm{ohms} / \mathrm{square}$. In the samples irradiated to doses higher than the threshold dose $\left(2.5 D_{\text {th }}\right)$ the isolation persists up to the temperature of $350{ }^{\circ} \mathrm{C}$. The annealing characteristics in this case are similar to those of threshold dose, with slightly better sheet resistivity at lower annealing temperatures. The increase of the irradiation dose to $10 D_{\text {th }}$ leads to the decrease of $R_{\mathrm{s}}$ because of hopping conduction in the highly damaged region. During post irradiation annealing there is a progressive increase of $R_{\mathrm{s}}$ with increasing temperature up to $300^{\circ} \mathrm{C}$, since the carrier hopping becomes less effective as the damage is repaired. The defects are removed by annealing above this temperature, which would correspond to the annealing of defect clusters or defect complexes. Figure 3 presents the evolution of $R_{s}$ with the annealing temperature of the implanted $n$-type resistors using four different doses: $2 \times 10^{15} \mathrm{~cm}^{-2}\left(D_{\mathrm{th}}\right)$; $5 \times 10^{13} \mathrm{~cm}^{-2}\left(0.025 D_{\mathrm{th}}\right) ; 2 \times 10^{14} \mathrm{~cm}^{-2}\left(0.1 D_{\mathrm{th}}\right)$, and $5 \times 10^{14} \mathrm{~cm}^{2}\left(0.25 D_{\mathrm{th}}\right)$. In the sample irradiated with the lowest dose $0.025 D_{\text {th }}, R_{\mathrm{s}}$ starts to decrease after $200^{\circ} \mathrm{C}$ after achieving a maximum value close to $10^{8} \mathrm{ohms} / \mathrm{square}$. Increasing the dose to $0.1 D_{\text {th }}$ the isolation persists up to $300{ }^{\circ} \mathrm{C}$. The resistivity is reduced by about 3 to 5 orders of magnitude during annealing at $400-550^{\circ} \mathrm{C}$. In the sample irradiated to the dose of $0.25 D_{\text {th }}$ one can observe a similar behaviour of the evolution of sheet resistivity with annealing temperatures as in the case of 2.5 times smaller dose. The isolation is stable up to $300^{\circ} \mathrm{C}$. After an irradiation to the threshold dose of $2 \times 10^{15} \mathrm{~cm}^{-2}$, higher sheet resistivity values are stable even after annealing at $500^{\circ} \mathrm{C}$. It is interesting to note in Fig. 3 that resistors irradiated with threshold doses provide a large thermally stable process window. Figure 3 also explains that the devices of starting material with high sheet carrier concentration are effectively isolated with threshold doses. Further higher doses, for example in the case of figure 2, are not needed to obtain better isolation. The larger thermally stable window (till $500^{\circ} \mathrm{C}$ ) achieved in the case of the results shown in figure 3 , is sufficient for subsequent processing such as contact sintering.

\section{Summary:}

The thermal stability of electrical isolation is found to depend on the original free carrier concentration and the ion dose. A significant improvement in the thermal stability is obtained in samples irradiated to a dose we call a threshold dose, $D_{\mathrm{th}}$, for higher initial sheet carrier concentrations. In these samples the isolation is maintained up to $500{ }^{\circ} \mathrm{C}$, which seems adequate for most technological applications. Optical measurements indicate that the depths to which the optical changes extend in the as-implanted devices are greatest for liquid nitrogen implantation where no dynamic annealing occurs. Optical effects are also dose dependent. 
The data presented here shows that both electrical and optical effects are important to consider when choosing different implantation parameters for an efficient device isolation scheme.

\section{Acknowledgements:}

The authors gratefully acknowledge financial support from the UK Engineering and Physical Sciences Research Council (EPSRC).

\section{References:}

[1] H. Boudinov, A. V. Coelho, and J. P. DeSouza, J. Appl. Phys. 91, 6585 (2002).

[2] S. J. Pearton, Mater. Sci. Rep. 4, 313 (1990).

[3] I. Danilov, J. P. DeSouza, and H. Boudinov, J. Appl. Phys. 92, 4261 (2002).

[4] M. A. Mentzer, R. G. Hunsperger, J. M. Zavada, H. A. Jenkinson, and T. J. Gavanis, Appl. Phys. A. 32 , 19 (1983)

[5] R. G. Hunsperger. Integrated Optics: Theory and Technology (Springer, Berlin, 1982).

[6] T. S. Moss. Optical properties of Semiconductors (Butterworths, London, 1959).

[7] S. Somekh, E. Garmire, A. Yariv, H. L. Garvin, R. G. Hunsperger, Appl. Opt. 13, 327 (1974).

[8] S. Ahmed, B. J. Sealy, and R. Gwilliam. IEEE Technical Digest: Electron Devices for Microwave and Optoelectronic Applications, 18 (2002).

[9] J. P. deSouza, I. Danilov, and H. Boudinov, J. Appl. Phys. 81, 650 (1997).

[10] J. P. de Souza, I. Danilov, and H. Boudinov, Appl. Phys. Lett. 68, 535 (1996).

[11] J. F. Ziegler, J. P. Biersack, and U. Littmark. The Stopping and Range of Ions in Solids (Pergamon, Oxford, 1985).

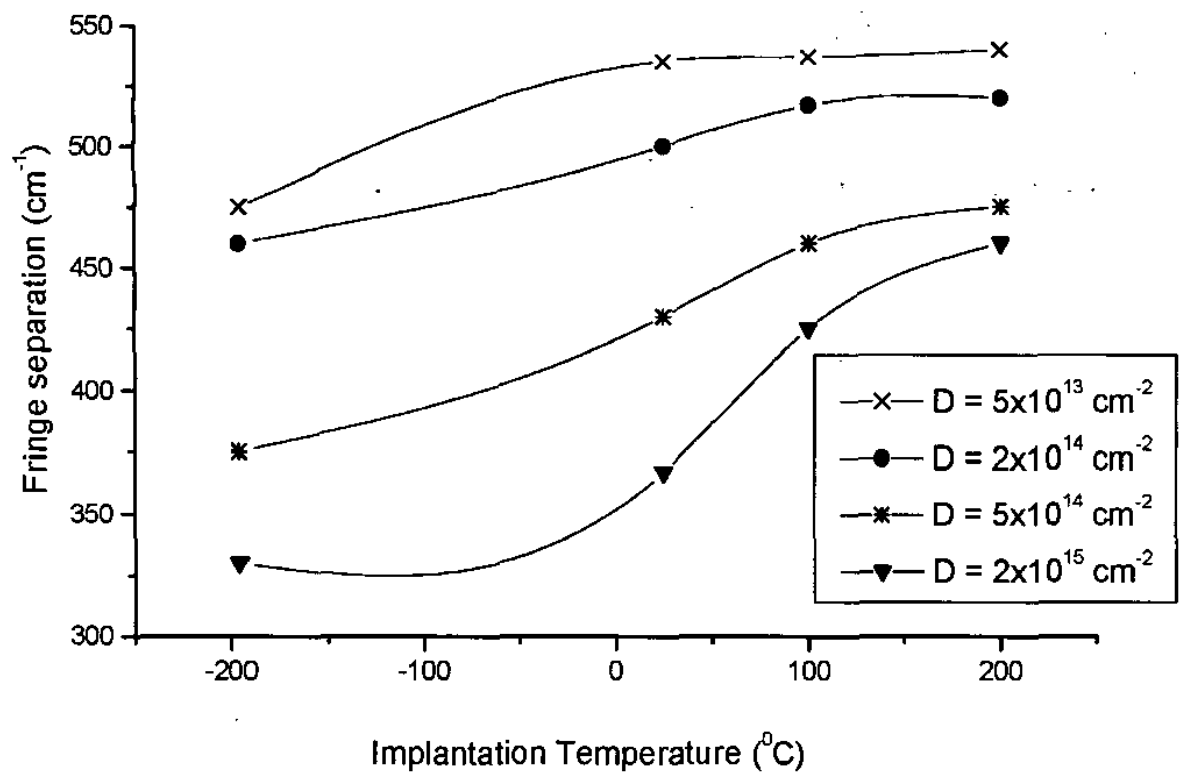

Figure 1: Infrared Reflectivity Measurements: Average Fringe Separation as a function of implantation temperature for proton-isolated GaAs layers. The average spectral separation is inversely proportional to the depths to which the optical effects extend. 


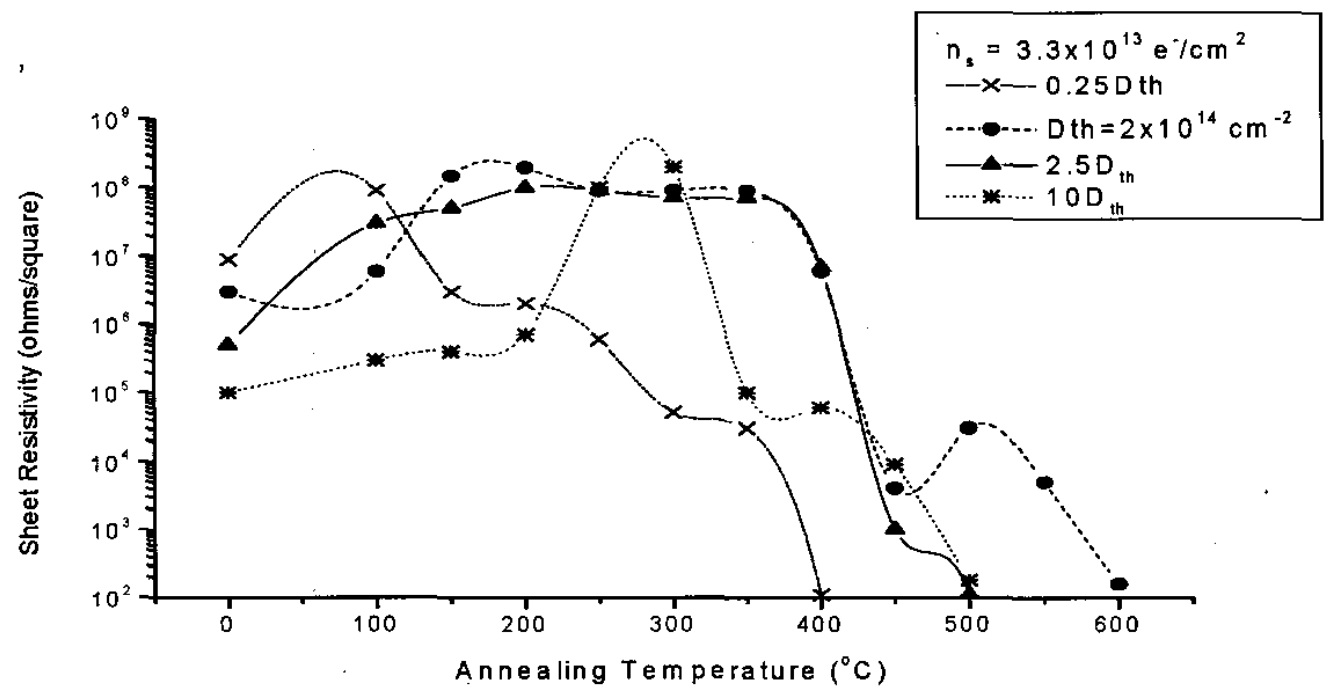

Figure2: Annealing stability of sheet resistance for layers implanted with $300 \mathrm{keV}$ protons at $\mathrm{RT}$ to variable doses. The effect of threshold doses on the annealing temperatures and thermal stability is also a function of initial sheet carrier concentration, which is $3.3 \times 10^{13} \mathrm{e}^{-1} / \mathrm{cm}^{2}$ in this case.

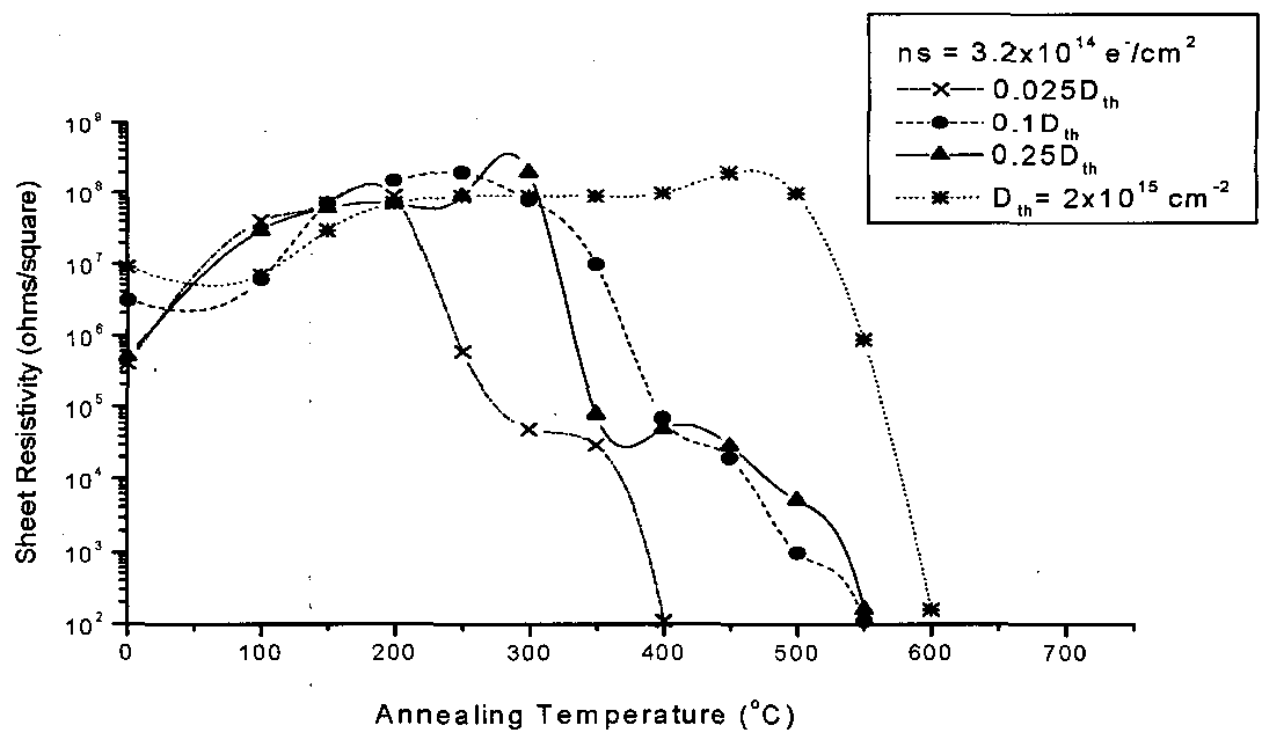

Figure3: Thermal stability of sheet resistivity for layers, implanted with $300 \mathrm{keV}$ protons at RT to variable doses. The effect of threshold doses on the annealing temperatures and thermal stability is also a function of initial sheet carrier concentration, which is $3.2 \times 10^{14} \mathrm{e}^{-1} / \mathrm{cm}^{2}$ in 
this case. The devices irradiated with threshold doses provide a large thermally stable process window. 